\title{
Enhancement of objects in photoacoustic tomography using selective filtering ${ }^{1}$
}

\author{
Dong-ho Shin ${ }^{\mathrm{a}}$, Yoonseok Yang ${ }^{\mathrm{b}}$ and Chul-Gyu Song, ${ }^{\mathrm{a}, \mathrm{c}^{*}}$ \\ ${ }^{a}$ Department of Electronic Engineering, Chonbuk National University, Jeonju-si, Jeonbuk, Korea \\ ${ }^{b}$ Division of Biomedical Engineering, Chonbuk National University, Jeonju-si, Jeonbuk, Korea \\ ${ }^{c}$ Center for Advanced Bioimaging Research, Chonbuk National University, Jeonju-si, Jeonbuk, Korea
}

\begin{abstract}
Here we developed a real-time photoacoustic tomography (PAT) imaging acquisition device based on the linear array transducer utilized on ultrasonic devices. Also, we produced a phantom including diverse contrast media and acquired PAT imaging as the light source wavelength was changing to see if the contrast media reacted. Indocyanine green showed the highest reaction around the $800-\mathrm{nm}$ band, methylene blue demonstrated the same in the $750-\mathrm{nm}$ band, and gold nanoparticle showed the same in the 700-nm band. However, in the case of superparamagnetic iron oxide, we observed not reaction within the wavelength bands used herein to obtain imaging. Moreover, we applied selective filtering to the acquired PAT imaging to remove noise from around and reinforce the object's area. Consequentially, we could see the object area in the imaging was effectively detected and the image noise was removed.
\end{abstract}

Keywords: Photoacoustic image, real-time, back projection algorithm, ultrasonic imaging, selective filtering

\section{Introduction}

Photoacoustic imaging is a technique that utilizes photoacoustic effects to noninvasively visualize biological tissues. A portion of the short electromagnetic pulse that is injected into a biological tissue is absorbed by the tissue. The absorbed energy is converted to heat, causing thermoelastic expansion and resulting in an ultrasonic release that has a wide range of frequency bands. The photoacoustic imaging technique involves restructuring of imaging based on collected ultrasonic information using such characteristics [1-3]. The photoacoustic imaging technique combines the benefits of both optical and ultrasonic imaging techniques. That is, the higher optical contrast ratio of optical imaging techniques such as optical coherence tomography (OCT) and effective spatial resolution ultrasonic imaging techniques can be realized simultaneously. Moreover, based on the difference of photoacoustic occurrence intensity according to the various light absorption rates of biological tissues, the biological changes can be visualized. As such, the photoacoustic imaging technique has been dynamically applied to diagnose diseases, such as skin, breast, and prostate cancers [4-6].

Studies are underway on real-time photoacoustic tomography techniques to more noninvasively

\footnotetext{
${ }^{1}$ Dong-ho Shin and Yoonseok Yang contributed equally to this work.

* Address for correspondence: Chul-Gyu Song, Electronic Engineering Department, Chonbuk National University, 66-14 Deok jin-dong, Jeonju-si, Jeonbuk, Korea. Tel.: +82 63270 4282; E-mail: song133436@gmail.com.
}

0959-2989/15/\$35.00 @ 2015 - IOS Press and the authors. 
acquire images of functioning biological tissue. Real-time photoacoustic imaging devices are being developed using an array transducer with multiple ultrasound transducers, and a two-dimensional array transducer-based imaging acquisition photoacoustic imaging system was recently announced that overcame the limits of the linear array transducer. The development of these real-time photoacoustic imaging methods has been actively researched by Professor Lihong Wang's research team at Washington University $[7,8]$.

Photoacoustic imaging could be exposed to noises in various ways. Noise can arise due to the imaging device's internal sound, limited detector sensitivity and size, and the size assumed by an image reconstruction algorithm. These noises complicate the interpretation of the acquired photoacoustic images. Accordingly, noise elimination is essential for the process. In this context, studies are investigating methods of removing noise from photoacoustic images. Moock, et al. proposed a method to filter photoacoustic signals then reconstruct images to this end [9], and Ahang, et al. improved images by applying the Back-Projection algorithm first for reconstruction and then using the anisotropic diffusion method [10]. Oruganti, et al. emphasized the blood vessel structure by applying the vessel filtering technique to blood vessel structure images gained in three-dimensional photo acoustic images [11]. However, noise elimination in photoacoustic images has not been actively researched.

In this letter, we describe a real-time photoacoustic tomography (PAT) image acquisition system that uses the linear array transducer for ultrasonic devices. We also produced a phantom including diverse contrast media and obtained PAT images with changing laser wavelengths. Moreover, the acquired PAT images were processed by the selective filtering technique described herein to remove background noise and better detect the objects within the images.

\section{Materials and methods}

\subsection{Photoacoustic image acquisition system}

To acquire PAT images, laser light source radiation, photoacoustic signal obtaining, and image conversion should be synchronized. As such, in this study, we used a data acquisition (DAQ) device based on the PXI platform. The utilized PXI platform has a $50-\mathrm{MHz}$ sampling frequency, 12-bit resolution, and 128 analogue input channels and capable of communicating with a PC at $192 \mathrm{MB} / \mathrm{s}$ speed based on the direct memory access method. The DAQ's sample clock frequency range was from $25 \mathrm{MHz}$ to $50 \mathrm{MHz}^{2}$, maximum input voltage swing of $2 \mathrm{v}_{\mathrm{pp}}$ differential and $1 \mathrm{v}_{\mathrm{pp}}$ for single-ended, the gain range was from $-5 \mathrm{~dB}$ to $31 \mathrm{~dB}$ and gain resolution is $0.125 \mathrm{~dB}$. To deliver a trigger signal that marks the status and time points of light source radiation, we made our own laser trigger controller and used the linear array probe consisting of 128 transducer-centric frequencies around $5 \mathrm{MHz}$ to measure the photoacoustic signals. To amplify the measured photoacoustic signals and their filtering, we produced a 128 -channel preamplifier that has $40-\mathrm{dB}$ benefits and a $5-\mathrm{MHz}$ pass band and used it in front of the DAQ. Figure 1 shows the structure of the real-time PAT image acquisition system.

\subsection{Photoacoustic image acquisition system}

The back projection algorithm was employed to reconstruct the images based on data acquired from the PAT image acquisition system. The back projection algorithm, which consists of the back projection of the transmission of the photoacoustic image over time that is used to reconstruct the 
initial photoacoustic distribution, is widely used for photoacoustic imaging. Eqs. (1) and (2)

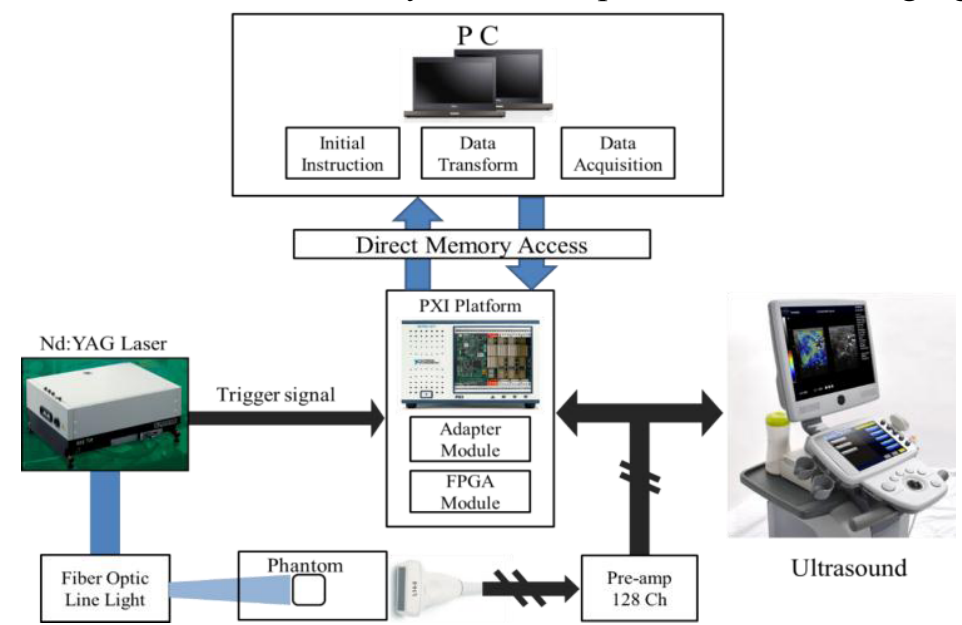

Fig. 1. Structure of the real-time photoacoustic tomography image acquisition system.

demonstrate the back projection algorithm [12].

$$
\begin{gathered}
p_{0}=(\vec{r})=\int_{\Omega_{0}} \frac{d \Omega_{0}}{\Omega_{0}}\left[2 p\left(\vec{r}_{0}, v_{s} t\right)\right], t=\left|\vec{r}-\frac{\vec{r}_{0}}{v_{s}}\right| \\
d \Omega_{0}=\frac{d S_{0}}{\left|\vec{r}-\vec{r}_{0}\right|^{2}} \bullet \frac{\hat{n}_{0}^{s} \cdot\left(\vec{r}-\vec{r}_{0}\right)}{\left|\vec{r}-\vec{r}_{0}\right|}
\end{gathered}
$$

Where, $p_{0}$ represents the initial photoacoustic signal, $\vec{r}$ represents a vector for a coordinate of the desired image, and $\vec{r}_{0}$ represents a coordinate of the measurement of a transducer. $v_{s}$ represents the speed of ultrasonic waves, $p\left(\vec{r}_{0}, v_{s} t\right)$ represents the measured photoacoustic signals, and $\Omega_{0}$ represents a solid angle in the $\vec{r}$ direction of the desired image on the surface.

\subsection{Phantom experiments}

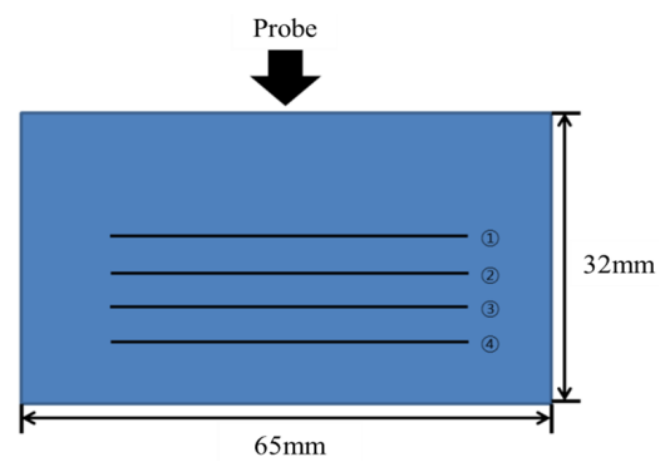

Fig. 2. Different phantom layer for PAT images. (1) Methylene blue, (2) Indocyanine green, (3) gold nanoparticle,(4) superparamagnetic iron oxide. 
We produced a phantom using the real-time PAT image acquisition device for PAT images. The phantom was made of gelatin (protein, $84-90 \%$; water, $8-12 \%$ ) to create an environment similar to that of biological tissues in a $65 \times 32 \times 21 \mathrm{~mm}$ hexahedron shape. Figure 2 shows the phantom. The

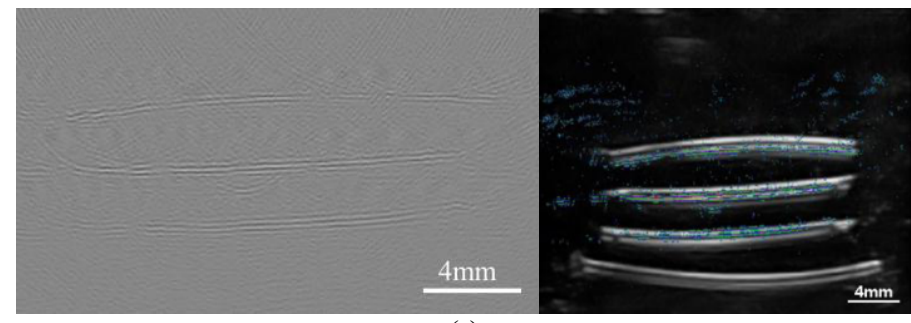

(a)

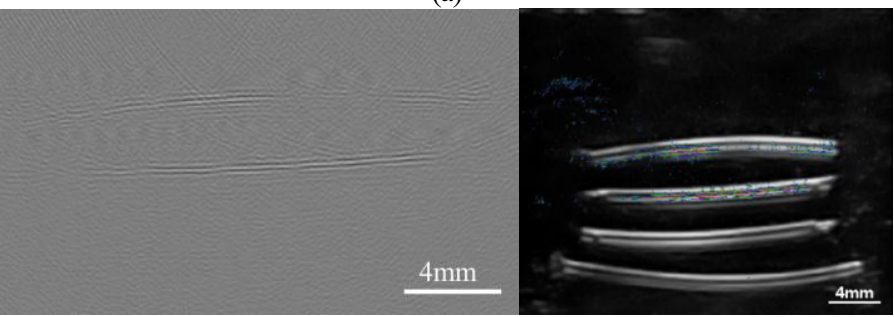

(b)

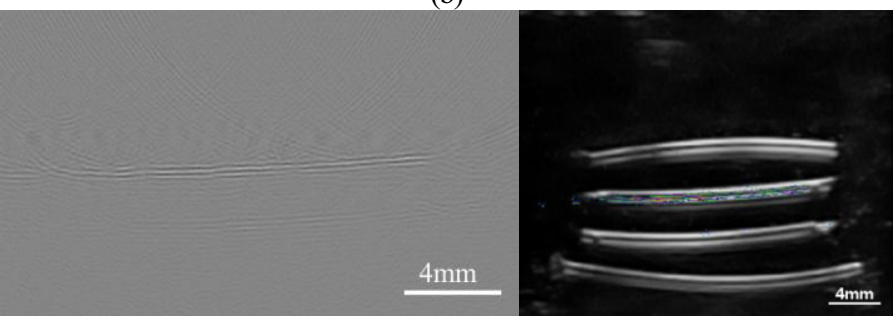

(c)

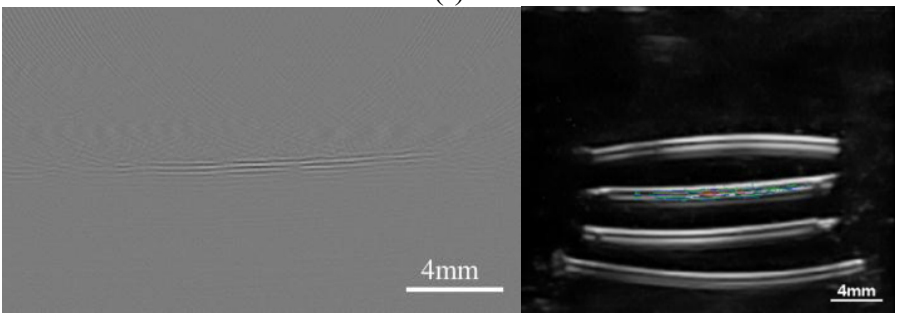

(d)

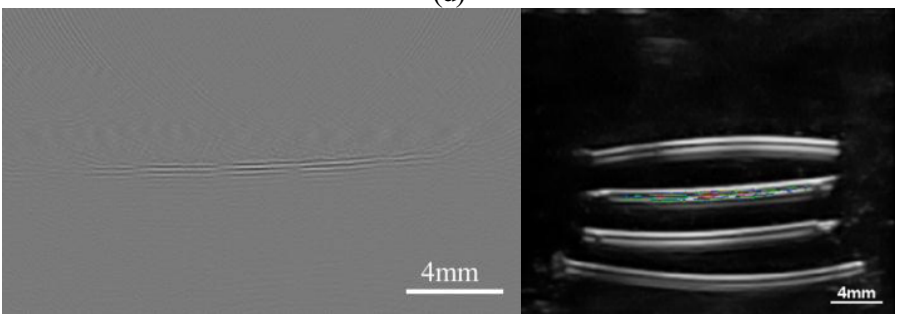

(e)

Fig. 3. Acquired PAT image of the phantom on the left side and the overlay image of PAT and ultrasound on right. (a) Image based on a 700-nm wavelength laser light source. (b) Image based on a 750-nm wavelength laser light source. (c) Image based on an 800-nm wavelength laser light source. (d) Image based on an 850-nm wavelength laser light source. (e) Image based on a $900-\mathrm{nm}$ wavelength laser light source. 
phantom was injected using a polymer tube with an internal diameter of $0.5 \mathrm{~mm}$ and an external diameter of $0.7 \mathrm{~mm}$ that contains four different contrast media. This configuration enables checking of the contrast media's reaction according to laser light source wavelengths. The contrast media injected in each tube include: methylene blue, indocyanine green, gold nanoparticle, and superparamagnetic iron oxide (SPIO). We obtained the images using the phantom and the real-time PAT image acquisition device. A Nd:YAG Q-switched laser (NT352-A20-AW, EKSPLA) was used as the light source. We changed its wavelength from $700 \mathrm{~nm}$ to $900 \mathrm{~nm}$ in $50-\mathrm{nm}$ increments with a $5-\mathrm{Hz}$ radiation speed and the laser power of $30 \mathrm{~J}$. The beam size on to the sample was $2 \mathrm{~cm}$ approximately. The imaging resolution is $0.03 \mathrm{~mm}$ and also the imaging depth is $5 \mathrm{~cm}$. As the phantom measurement scope is far wider than the beam shape of the light source, a fiber optic line light (NT57-019, Edmund Optics) was used to diffuse the light source under investigation. Figure 3 shows the PAT images of the phantom acquired by the produced PAT image acquisition device and the overlaid images with the ultrasound image and the PAT image. The acquired image is $488 \times 400$ pixels with $0.08 \mathrm{~mm} \times 0.08$ $\mathrm{mm}$ resolution per pixel.

\section{Result and discussion}

Looking at the acquired PAT images, we observed a tube containing methylene blue, indocyanine green, and gold nanoparticle in the PAT image of the $700-\mathrm{nm}$ wavelength light source and a tube containing methylene blue and indocyanine green in the $750-\mathrm{nm}$ wavelength image. A tube containing

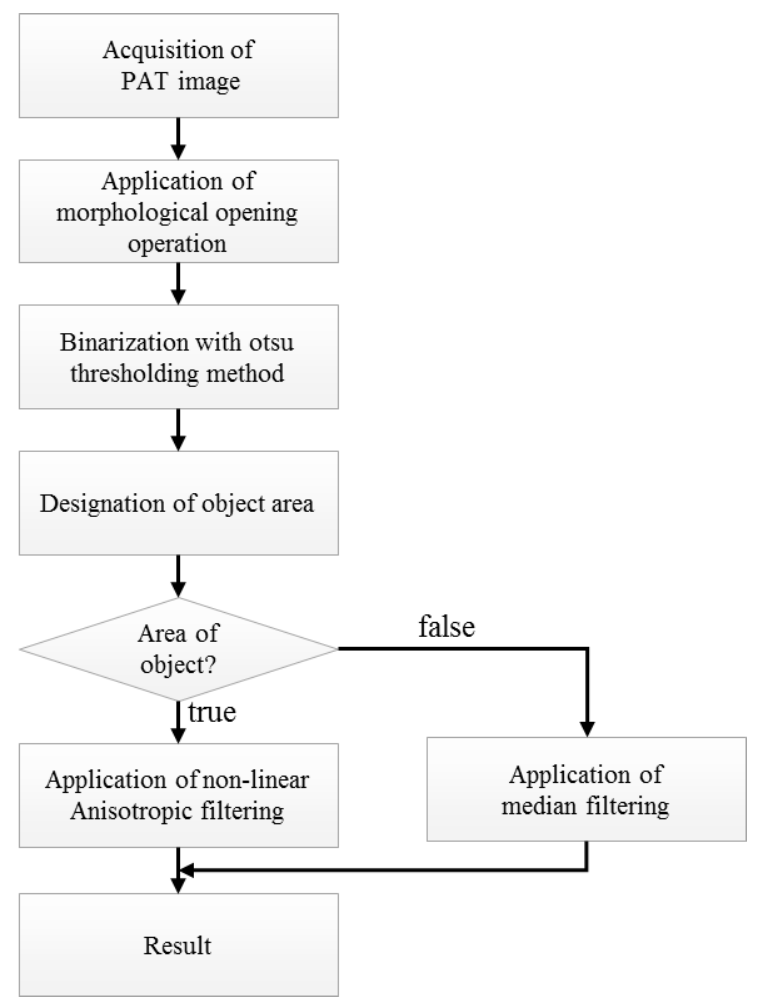

Fig. 4. Flowchart of the selective filtering method. 
indocyanine green only was observed in the $800-\mathrm{nm}$ wavelength image. This finding means that methylene blue is reactive only under the $750-\mathrm{nm}$ wavelength, while the gold nanoparticle is reactive only under the 700-nm wavelength. In particular, indocyanine green reacted at all wavelength bands between 700 and $900 \mathrm{~nm}$, while the best reaction was seen at $800 \mathrm{~nm}$. However, the laser power was $30 \mathrm{~mJ}$ for all the conditions and SPIO had no reaction at all in the laser light source wavelengths used herein. In this letter, selective filtering technique was applied to reinforce the area of the object in the acquired PAT image. The selective filtering technique removes noises within the images and emphasizes the area of an image object. It also divides image data area into objects and backgrounds and applies different filtering methods to each area to emphasize the object area. Figure 4 shows a flowchart of the selective filtering method.

The morphology opening operation that is often used in selective filtering extracts the characteristics of an object area inside images by applying a sequential erosion and dilation operation. At this point, the area's shape is controlled depending on the structural component [13]. Otsu's thresholding method was used for the image binarization. The proposed method by Otsu is an algorithm that automatically determines the threshold via a statistical method based on the image histogram [14].

Nonlinear anisotropic filtering and median filtering were also adopted in the image filtering process. Nonlinear anisotropic filtering in particular removes noise from both the general areas and the edge components to improve the image by tracing the direction of the edge information within the image to select the optimal direction [15].

The PAT image which was taken at $700 \mathrm{~nm}$ wavelength was selected for the selective filtering and can be seen in Figure 5(a). In the next step of the selective filtering a disked shaped structuring element is chosen and morphological opening operation is applied to the input image, the output after this process is shown in Figure 5(b). Later the threshold used for image binarization and the result is shown in Figure 5(c). The value of the threshold is acquired by the Otsu's thresholding method and then multiplied by an experimental coefficient 0.85 . The final image after the filtering method shown in Figure 5(d), which has gone through the filtering operation as follows, the noise is removed by the use of a nonlinear anisotropic filter in the object area and the use of a median filter in the non-object

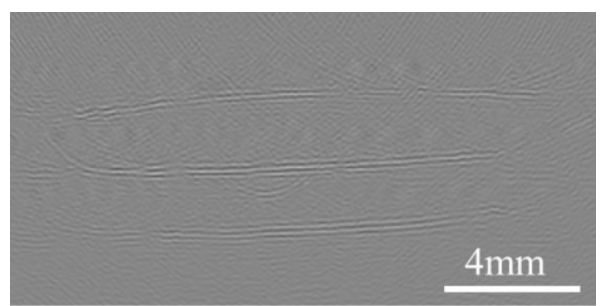

(a)

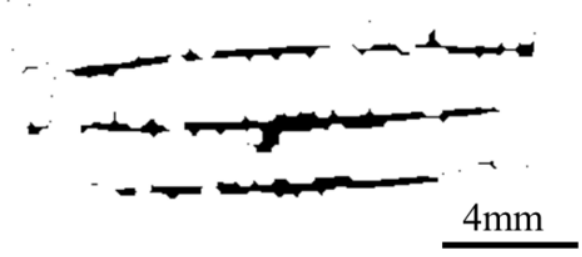

(c)

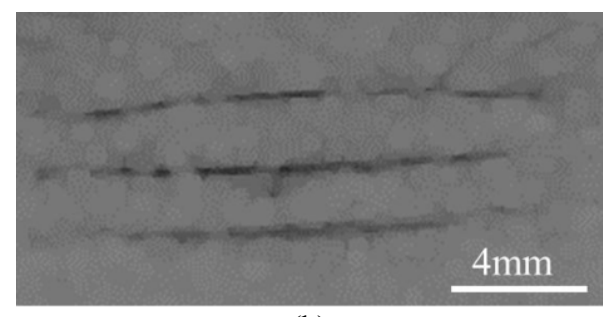

(b)

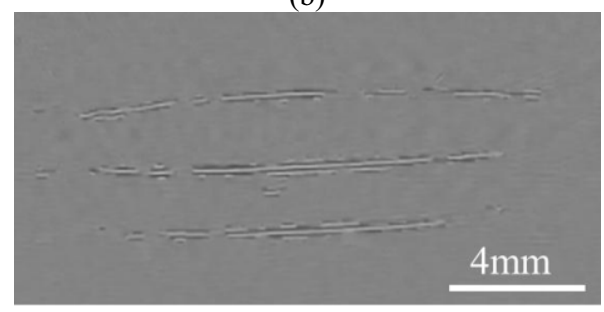

(d)

Fig. 5. Results of applying selective filtering. (a) Original image. (b) Result of applying morphological opening operation. (c) Result of image binarization. (d) Result of image filtering. 
Table 1

Quantitative comparison of the photoacoustic tomography image filtering result

\begin{tabular}{clll}
\hline & $\begin{array}{l}\text { Mean } \\
\text { filtering }\end{array}$ & $\begin{array}{l}\text { Median } \\
\text { filtering }\end{array}$ & $\begin{array}{l}\text { Selective } \\
\text { filtering }\end{array}$ \\
\hline RMSE & 8.810 & 8.997 & 7.191 \\
\hline PSNR $(\mathrm{dB})$ & 29.535 & 29.348 & 31.395 \\
\hline MAE & 5.343 & 5.378 & 4.978 \\
\hline Note: RMSE, root mean square error; PSNR, peak signal-to-noise ratio; MAE, mean absolute error
\end{tabular}

areas to emphasize the images. As a result, the acquired PAT images' objects were more clearly expressed and the neighboring noises were eliminated. However, the morphological opening operation demonstrated that the objects failed to keep their original shape. This seems to be due to the shape of the structural components used in the morphological opening operation. We applied selective, median, and mean filtering to the PAT images and calculated the average root mean square error, peak signal to noise, and mean absolute error. Table 1 shows the quantitative data of the filtering results of the acquired PAT image.

\section{Conclusion}

In this letter, we described our creation of a real-time PAT image acquisition device based on a linear array transducer used in ultrasonic devices. We also produced a phantom containing diverse contrast media and observed the contrast media reactions by acquiring PAT images under changing light source wavelengths. As a result, we found that indocyanine green was reactive throughout the wavelength bands used herein for image acquisition and peaked at the $800-\mathrm{nm}$ band. Methylene blue was found to be reactive in wave bands not exceeding $750 \mathrm{~nm}$, while gold nanoparticle was reactive at wave bands not exceeding $700 \mathrm{~nm}$. However, SPIO was non-reactive at all of the wavelength bands used herein for image acquisition. Moreover, the acquired PAT images were processed with selective filtering to remove noise and emphasize the object area. Consequently, the detected object area failed to maintain its original shape. This is thought to be attributable to the shapes of the structural components used in the morphological opening operation. Therefore, in a subsequent study, we plan to conduct more diversified experiments on contrast media and light source wavelength bands to identify optimal wavelengths for specific contrast media and explore ways to maintain object area shape by improving the selective filtering technique

\section{Acknowledgment}

This paper was supported by research funds of Chonbuk National University in 2013.

\section{References}

[1] A.A. Oraevsky and A.A. Karabutov, Optoacoustic tomography, Biomedical Photonics Handbook 34 (2003), 1-34.

[2] L.V. Wang and H. Wu, Biomedical Optics: Principles and Imaging, Wiley, Hoboken, New Jersey, 2007.

[3] L.V. Wang, Photoacoustic Imaging and Spectroscopy, 1st ed., CRC Press, Boca Raton, Florida, 2009.

[4] Oh Jung-Taek, et al., Three-dimensional imaging of skin melanoma in vivo by dual-wavelength photoacoustic microscopy, Journal of Biomedical Optics 11.3 (2006), 034032-034032. 
[5] S. Manohar, A. Kharine, J.C. van Hespen, W. Steenbergen and T.G. van Leeuwen, The twente photoacoustic mammoscope: System overview and performance, Physics in Medicine and Biology 50.11 (2005), 2543-2557.

[6] Mohammad A. Yaseen, et al., Hybrid optoacoustic and ultrasonic imaging system for detection of prostate malignancies, Biomedical Optics (BiOS), International Society for Optics and Photonics, 2008, pp. 68560T-68560T.

[7] Qiuzhen Zhou, Xuanrong Ji and Da Xing, Full-field 3D photoacoustic imaging based on plane transducer array and spatial phase-controlled algorithm, Medical Physics 38.3 (2011), 1561-1566.

[8] John Gamelin, et al., A real-time photoacoustic tomography system for small animals, Optics Express 17.13 (2009), 10489-10498.

[9] V. Moock, et al., Signal processing for photoacoustic tomography, 2012 5th International Congress on Image and Signal Processing (CISP), Chongqing, China, 2012, pp. 957-961.

[10] Y. Zhang and Y. Wang, An improved filtered back-projection algorithm for photoacoustic tomography, 5th International Conference on Bioinformatics and Biomedical Engineering (iCBBE), Wuhan, China, 2011, pp. 1-4.

[11] T. Oruganti, J.G. Laufer and B.E. Treeby, Vessel filtering of photoacoustic images, International Society for Optics and Photonics, San Francisco, 2013, pp. 85811W-85811W.

[12] M. Xu and L.V. Wang, Universal back-projection algorithm for photoacoustic computed tomography, Biomedical Optics, International Society for Optics and Photonics, San Jose, 2005, pp. 251-254.

[13] R.C. Gonzalez, R.E. Woods and S.L. Eddins, Morphological Image Processing, in: Digital Image Processing Using Matlab, Pearson Prantice Hall, Upper Saddle River, New Jersey, 2004.

[14] Otsu Nobuyuki, A threshold selection method from gray-level histograms, IEEE Transactions on Systems, Man and Cybernetics 9 (1979), 62-66.

[15] Maciej M. Orkisz, et al., Improved vessel visualization in MR angiography by nonlinear anisotropic filtering, Magnetic Resonance in Medicine 37.6 (1997), 914-919. 